\title{
Venus news trap
}

\author{
The detection of phosphine in the cloud decks of Venus generated 4,700 news stories around the world. This kind \\ of coordination was only possible because of our embargo policy.
}

$\mathrm{R}$ ecently, you may have noticed media reports on the detection of phosphine at Venus. The paper was covered by 62 major news outlets in print, online and on television. If nothing else, the news of the discovery was a momentary welcome respite from stories of the pandemic; wildfires, floods and tropical storms due to the climate emergency; plagues of locusts; police brutality; and mass protests against social and economic inequality - the list goes on, seemingly on repeat.

The presence of phosphine in the atmosphere of Venus might be akin to the presence of methane on Mars, with both gases being potential biomarkers demanding independent verification, as explained in this article. On Earth, phosphine is associated with anaerobic organisms. It is also found at Jupiter, where it is produced in the hot, high-pressure atmospheric layers deep down and gets churned up into the upper atmosphere. As Venus lacks the energy to form and sustain the detected levels of phosphine through a known channel, some unexpected process in the atmosphere, surface or subsurface must be maintaining its presence in the clouds. It may be that our understanding of phosphine chemistry is incomplete; indeed, there is no information on the photochemistry of the cloud droplets. Thus unexplained chemistry would seem a plausible explanation. But the possibility of life - even the microbial kind - in the hyperacidic environment of the clouds of Venus was far too exciting for most news outlets to resist.

As with all papers published by Nature Research, detailed information of the paper was sent by our press office under embargo to thousands of registered journalists six days before the paper went live. Until the embargo lifts, upon publication, the information and documents are strictly confidential. This period provides journalists sufficient time to read the full paper, conduct background research, contact the authors for clarification or further explanation and share the information with experts (who must in turn respect the embargo) in order to obtain independent assessments and quotations. In this manner, our press office ensures that any registered

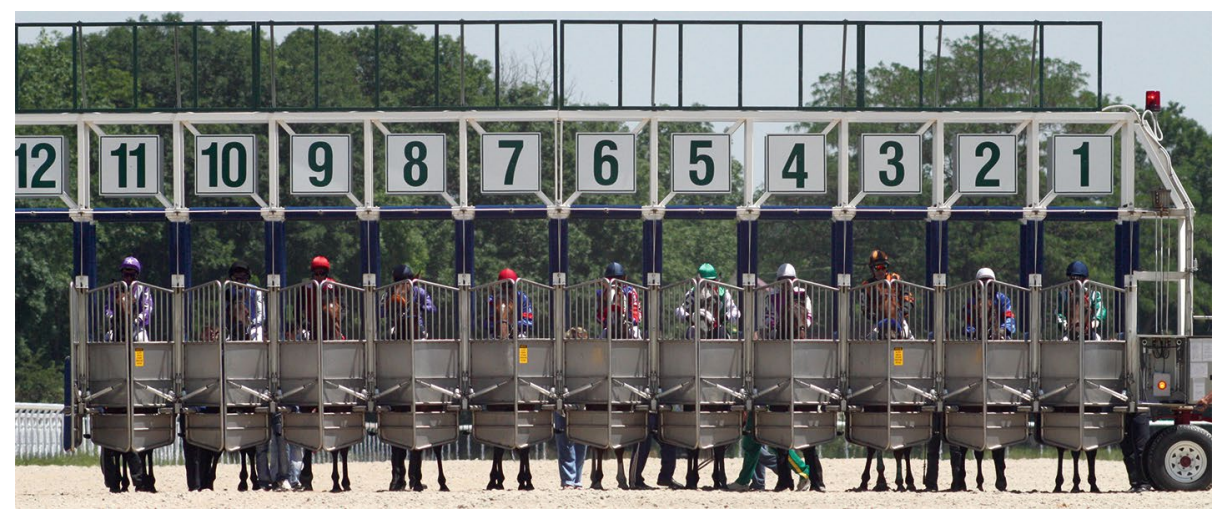

Credit: mikle15 / Alamy Stock Photo

media representative - from a journalist writing for a local newspaper to a news presenter at a major television channel who wishes to report a given result has all the materials they need at the same time, with equal access.

The embargo period also helps authors and their institutions plan their press releases and outreach activities, but it is not a blanket ban on scientific communication. Many people misinterpret our policy to mean that authors cannot discuss their results at conferences or post preprints on the arXiv. In fact, authors are free to talk about their research results with their colleagues, present them at conferences and post the submitted version of their paper on any preprint archive server at any time prior to publication, and indeed after it. More information on our preprint policy can be found here.

In addition, we have some guidelines for authors talking to journalists. Journalists often attend conferences and check on preprint archives, so they may well contact an author for comment. In such cases it is fine to answer questions that clarify or explain the research; however, journalists should bear in mind that until the paper has undergone peer review, there is always a risk that the conclusions might change. For this reason, we do not encourage authors to seek media coverage before the paper is finalized. Moreover, if there is any media attention prior to publication, we would take that into account when deciding whether to put out a press release ourselves. Hence, it is often better for all involved for authors to ask journalists to wait until that week before publication when all the materials will be available to everyone under embargo.

In the case of the phosphine paper, each of the institutions involved put out their own press release. There was also a press briefing arranged by the United Kingdom's Royal Astronomical Society to coincide with the lifting of the embargo, followed by opportunities to ask questions of the authors on Twitter and Reddit. This kind of concerted effort only works because of the embargo. However, there were some leaks prior to publication. One journalist even complained on Twitter that the embargo stopped them from addressing the swirling misinformation. But the whole point of the embargo is to ensure accurate and balanced coverage. The coordinated press releases allowed each institution to highlight their contribution to the global effort, with quotes from the scientists emphasizing that the source of phosphine in the clouds of Venus needs to be independently confirmed and its origins explored.

Published online: 8 October 2020 https://doi.org/10.1038/s41550-020-01240-9 